ARTICLE OPEN

\title{
Enhanced hydrological extremes in the western United States under global warming through the lens of water vapor wave activity
}

\author{
Jian Lu (iD) ${ }^{1}$, Daokai Xue ${ }^{2}$, Yang Gao ${ }^{3}$, Gang Chen ${ }^{4}$, L. R. Leung ${ }^{1}$ and P. Staten ${ }^{5}$
}

A novel diagnostic framework based on the wave activity of column integrated water vapor (CWV) is used to probe into the higher moments of the hydrological cycle with bearings on the extremes. Applying the CWV wave activity analysis to the historical and RCP8.5 scenario simulations by the CMIP5 models reveals a super Clausius-Clapeyron rate of increase in the wet vs. dry disparity of daily net precipitation due to the enhanced stirring length of wave activity at the poleward flank of the storm track, despite a decrease in the hydrological cycling rate (HCR) measured by the reciprocal of wave activity residence time. The local variant of CWV wave activity unravels the unique characteristics of atmospheric rivers (ARs) in terms of their transport function and locally enhanced mixing efficiency. Under RCP8.5, the local moist wave activity increases by $\sim 40 \%$ over the northeastern Pacific and western United States by the end of the 21 st century, indicating lengthening and more frequent landfalling ARs with a consequence of a $\sim 20 \%$ increase in the related hydrological extremes $(P-E)^{+}$in the west coast, despite a robust weakening of the local HCR. These results imply that the unusually wet winter the west coast just experienced in 2016/17 might be a harbinger of more frequent wet extremes in a warmer climate.

npj Climate and Atmospheric Science (2018)1:7; doi:10.1038/s41612-018-0017-9

\section{INTRODUCTION}

The winter of 2016/2017 marked one of the wettest winters for the western United States, with Nevada and California witnessing their wettest and second wettest winter ever on record, respectively. Although the western US experiences large interannual and interdecadal variability associated with climate modes such as $\mathrm{El}$ Niño-Southern Oscillation and Pacific Decadal Oscillation, the record-setting hydrological events could have an anthropogenic contribution as the atmospheric moisture holding capacity increases in a warmer climate, amplifying both the wet and dry extremes. ${ }^{1}$ Many studies ${ }^{2-6}$ have documented that future warming would lead to enhanced moisture transport by the atmospheric rivers (ARs), synoptic scale features responsible for concentrated supply of moisture from the tropics to the western $U^{7,8}$ causing more frequent and more intense precipitation extremes. In terms of precipitation percentiles and return periods, a consensus on the intensification of mid-to-high latitude hydrological extremes is beginning to emerge. ${ }^{9-13}$ However, most studies have only focused on the statistical and descriptive aspects of the hydrological extremes (e.g., ${ }^{14,15}$ ) or theories derived from idealized simulations (e.g., see ref. ${ }^{10}$ ). There is thus a critical need for a more rigorous diagnostic framework for the hydrological extremes, preferably with dynamical underpinnings.

Integrated globally, the atmosphere can be thought of as a "reservoir" of moisture, with precipitation acting as the moisture sink, evaporation acting as the moisture source, and the hydrological cycling rate $(\mathrm{HCR})$ of moisture determined by the ratio of the globally integrated water vapor to precipitation $(P)$ or evaporation $(E)$. As the climate warms, the water vapor content in the atmosphere increases following the Clausius-Clapeyron (CC) relation at a rate of $\sim 7 \%$ per ${ }^{\circ} \mathrm{C}$ warming. But the rate of $P$ increase is not commensurate with the $\mathrm{CC}$ rate, owing to the global energy constraint. ${ }^{16,17}$ As a result, the global HCR must decrease in a warming climate. ${ }^{18}$ However, the portrayal of the reservoir and the concept of the hydrological cycle cannot be carried over directly to a location fixed in space because a grid box is not a real physical boundary; and precipitation and column integrated water vapor (CWV) in a grid box are only loosely correlated (cf. Figure S1 and Fig. 1).

To overcome this limitation of the traditional Eulerian budget approach, here we adopt the concept of finite-amplitude wave activity (FAWA) and the related contour-following diagnostic framework $^{19-22}$ (see Box and Method for details) for examining the higher moments that characterize the variability and extremes of the hydrological cycle. The extension to local wave activity (LWA) is well-suited for portraying regional extremes, with important implications for our understanding of ARs. In essence, LWA allows the calculation of a budget for the moving "reservoirs" in the atmosphere. We implement the wave activity analysis for the meridional distribution of the total waviness of the CWV, as well as its local variant for regional hydrological extremes. This is achieved by using daily data for the historical (1976-2005) and future (2070-2099) periods simulated by 16 climate models that participated in the Coupled Model Intercomparison Project Phase

\footnotetext{
${ }^{1}$ Atmospheric Sciences and Global Change Division, Pacific Northwest National Laboratory, Richland, WA, USA; ${ }^{2}$ School of Atmospheric Sciences, Nanjing University, Nanjing, China; ${ }^{3}$ Key Laboratory of Marine Environment and Ecology, Ministry of Education of China, Ocean University of China, Qingdao, China; ${ }^{4}$ Department of Earth and Atmospheric

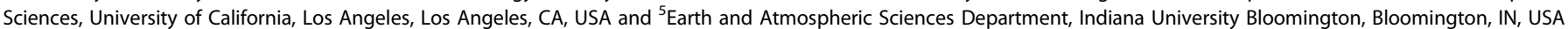
Correspondence: Jian Lu (Jian.Lu@pnnl.gov)

These authors contributed equally: Daokai Xue, Jian Lu.
}

Received: 8 June 2017 Revised: 9 October 2017 Accepted: 19 October 2017

Published online: 23 April 2018 
5 (CMIP5) ${ }^{23}$ under the historical forcing conditions and the RCP8.5 forcing scenario, respectively. A parsimonious recapitulation of the definition of CWV wave activity and the associated hydrological concepts are provided in text Box 1 for more casual readers. The more technical definition of CWV wave activity and derivation of the related budget and scaling are laid out in Method section.

\section{RESULTS}

A linear relationship between wave activity and its sink As in Lu et al., ${ }^{21}$ which analyzed aquaplanet simulations, a strong linear empirical relationship between the FAWA $(A)$ and the associated sink term $(P-E)$ also emerges in the comprehensive Coupled Model Intercomparison Project Phase 5 (CMIP5) models:

$$
(\widetilde{P-E}) \sim \frac{A}{\tau}
$$

where $\tau$ gives the residence time over which the sink acts to dissipate the wave activity anomalies. $\tau$ can be estimated accurately as the regression slope from the daily scatter plot between $A$ and $(P-E)$; the reciprocal of $\tau$ then provides a measure for the HCR. A similar relationship also holds for the local, moist wave activity $\left(A^{+}\right)$and its sink $(\widetilde{P-E})^{+}$(Fig. 1), arising from

\section{Box 1-A contour-following analysis for column water vapor}

The essence of the wave activity budget is a contour-following transformation of the traditional moisture budget (see $\mathrm{e}^{21}$ and Method for details). This allows areal integration over regions districted by contour $\mathrm{M}$ (black curve in inset Fig. 2a). The FAWA is the area-coordinate transformation on the CWV defined as the difference of the areal integral of $m$ over the moist areas minus that over the dry areas. Although constantly varying, the quasi-conservative $M$ contour serves effectively as the boundary of the moisture "reservoir" for budget analysis, with areas enclosed between the contour $\mathrm{M}$ and its corresponding equivalent latitude serving as a measure of the size of the "reservoir". The equivalent latitude $\left(\phi_{e}\right)$ is uniquely defined by the requirement that the areas bounded by the contour $M$ poleward and equatorward of $\phi_{e}$ are equal.

The sink of FAWA, $(P-E)$, excludes the zonal mean component of $P-E$ automatically by casting it onto the moist and dry sections of the CWV field, thus isolating the wave component of $P-E$. As such, $(P-E)$ represents the dry-vs.-wet disparity of the hydrological cycle. The advection of CWV by the rotational component of the moisture flux integrated along an $\mathrm{M}$ contour is identically zero (due to the Gauss divergence theorem). Hence the remaining budget terms all possess a tight linear relationship with the CWV wave activity itself, engendering a simple closure and more intuitive comprehension of the atmospheric moisture budget. $^{21}$ The resultant quantities from the area-coordinate transformation represent the second moments of the hydrological cycle, and their probability distributions become more Gaussian due to the central limit theorem. Thus, their mean and standard deviation capture the bulk of the information (to the extent of their proximity to Gaussian) one needs to know about the hydrological extremes.

The concept of wave activity can be extended to be longitude-dependent for studying local hydrological cycle. FAWA can thus be further decomposed into the parts associated with the northward moist intrusion $\left(A^{+}\right.$, blue area) and the southward dry intrusion $\left(A^{-}\right.$, brown area) and the corresponding sink terms are $(P-E)^{+}$and $(P-E)^{-}$, respectively (Fig. 2a). As it turns out, $A^{+}$and $(P-E)^{+}$are ideal metrics for describing moisture anomaly features such as $\mathrm{ARs}^{45}$ or moisture conveyor belts ${ }^{46,47}$ embedded in the moist section of cyclones. For a moist intrusion associated with an $A R$, the flux $\overline{v m}$ from the base at the equivalent latitude may be thought of as the "headwater"' of the AR. Furthermore, a tight empirical relationship holds between wave activity and its sink:

$$
\widetilde{(P-E)^{+}} \approx \frac{\left(A^{+}-A_{c}^{+}\right)}{T},
$$

as epitomized by Fig. 1. Here, $A_{c}^{+}$measures the CWV disturbance allowed prior to the occurrence of condensation or surface evaporation, representing the holding capacity of the CWV LWA. Through the scaling of the wave activity, $A_{c}^{+} \sim-\frac{1}{2} \eta_{c}^{+2} \frac{\partial M}{\partial y}$, with $\eta_{c}^{+}$assuming a meaning of "mean free path" of the air parcels within which the air parcels can travel freely poleward with the humidity content conserved. (B1) offers a new angle to interpret the hydrological cycle in the moist areas: $P-E$ is operating to restore the CWV towards the capacity level $A_{c}^{+}$at time scale $\tau$ (Fig. 2b). Since only the $A^{+}-A_{c}^{+}$portion (blue portion in Fig. $2 \mathrm{~b}, \mathrm{c})$ of $A^{+}$participates in the hydrological cycle and is subject to the dissipation by $P-E$, a $\mathrm{PR} \frac{A^{+}-A_{c}^{P}}{A^{+}}$can be defined to describe the hydrological cycle in the moist area (Fig. 2C). the fact that $(\widetilde{P-E})^{+}$can be approximated by the advection of the anomalous CWV (defined as $m^{\prime}=m-M$ ), i.e., ${\overline{v m^{\prime}}}^{+}$, if integrated over the full life cycle of a moist intrusion (or phase integral), with the latter being scaled as the wave activity $A^{+}$ divided by a time scale (see the Appendix of ${ }^{21}$ for the reasoning for this relationship). This tight relationship between the LWA of CWV and the local sink enables analysis of the local hydrological cycle and serves as a foundation for understanding the regional hydrological extremes. The ubiquity of the strong correlation between $A^{+}$and $(\widetilde{P-E})^{+}$in the midlatitude during boreal winter is demonstrated in Figure S2. Since $(\widetilde{P-E})^{+}$captures the higher moment of the hydrological cycle variability than the mean of $P$ $-E$, it has more immediate bearings on the hydrological extremes. This is evidenced by the spatial correspondence between $(\widetilde{P-E})^{+}$ and the 99.9th percentile of $P-E$ in both climatological mean and the response to climate change forcing (Fig. 3).

Figure 1 showcases the tight linear relationship between daily time series of wave activity $\left(A^{+}\right)$and $(P-E)^{+}$over the west coast of the US (averaged over the box in Fig. 4a) computed based on the historical simulation (1976-2005) and future RCP8.5 simulation (2070-2099) from the NCAR CCSM4 climate model. Remarkably, both wave activity and the associated sink are nearly normally distributed (side curves in Fig. 1) and both their mean and standard deviation increase in the future warming scenario, signifying more extreme moist events over the area. Similar increases are also found for the wave activity and $\left(\widetilde{P-E)^{+}}\right.$ averaged over the west coast of Europe $\left(35^{\circ}-55^{\circ} \mathrm{N}, 345^{\circ}-360^{\circ} \mathrm{E}\right)$ (Figure S3).

Future hydrological cycle changes: increased wet vs. dry disparity, enhanced wave stirring, and reduced HCR

Under climate change scenario RCP8.5, the winter wet vs. dry disparity $(\widetilde{P-E})$ increases almost everywhere in the Northern Hemisphere (Fig. 5a). Notably, the magnitude of increase varies considerably between latitudes, with a super CC peak ( 40\%) poleward of the mean storm track, consistent with a poleward shift of the zonal mean circulation. ${ }^{24-26}$ Making use of the linear relationship between $A$ and $(\widetilde{P-E})$, the fractional change of the latter can be scaled (see Eq. (5) in Methods) as the sum of the fractional change in hydrological cycle rate $\left(\tau^{-1}\right.$, Fig. 5e) and $A$ (Fig. $5 b)$, with the latter being further factored into the change of the Lagrangian meridional CWV gradient $\left(\frac{d M}{d y}\right.$, thermodynamical factor, Fig. 5d) and twice the fractional change of the wave stirring length $\left(\eta\right.$, dynamical factor, Fig. 5c) ${ }^{21}$ (see also Methods). Apparently, the dynamical factor is dominant in setting the meridional structure of the change of $(\widetilde{P-E})$ in most of the Northern Hemisphere; this effect is further verified by the fractional change of the (normalized) squared equivalent length $l_{e}^{2}$, which represents the enhancement of mixing due to the resolved stirring/stretching (see Methods for further explanation) and is directly estimated using the formula in Methods. Intriguingly, the HCR $\left(\tau^{-1}\right)$ tends to decrease everywhere in the $\mathrm{NH}$. Thus, for a given moisture disturbance, it takes longer for $P-E$ to dissipate it, meaning that the related processes for $P-E$ formation becomes less efficient with climate warming, possibly due to weakening of vertical motion. This seems to be in keeping with the finding that the work output from the atmospheric heat engine (and hence the driving for the atmospheric motion) is reduced with anthropogenic warming. ${ }^{27}$ Further analysis of the 6-hourly data from CESM Large Ensemble Project $^{28}$ indicates that the vertical structure of the lowertropospheric convergence extends upward (Jessie Norris, personal communication) and becomes less engaged with moisture, consistent with the upward shift of the atmospheric circulation under climate warming (e.g., ref. ${ }^{29}$ ). 


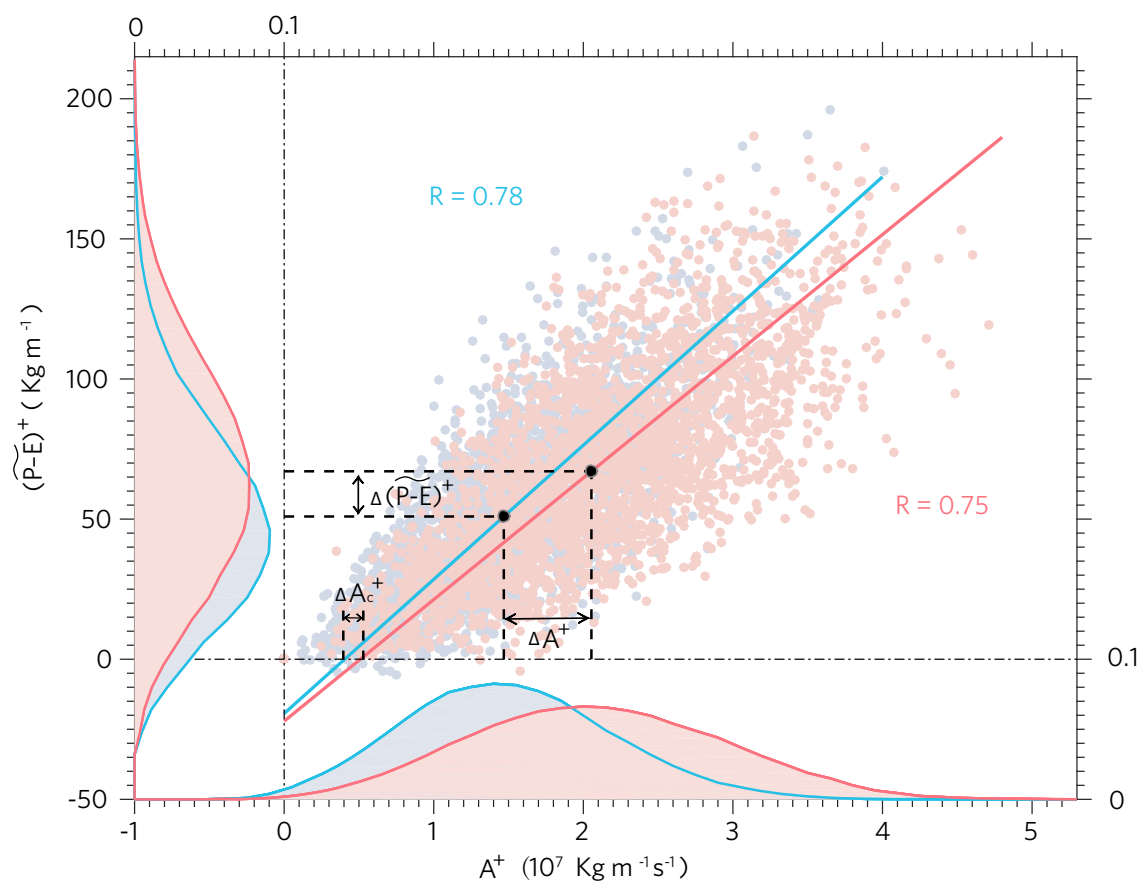

Fig. 1 Linear empirical relationship between $A^{+}$and $\left.\widetilde{(P-E}\right)^{+}$. The scatter of daily $A^{+}$against $\left.\widetilde{P-E}\right)^{+}$averaged over the western US $\left(30^{\circ}-50^{\circ}\right.$ $\mathrm{N}, 235^{\circ}-245^{\circ} \mathrm{E}$ ) and the corresponding marginal PDFs (curves) from the historical (blue) and future RCP8.5 (red) simulations by NCAR CCSM4. The intercept with the $y$-axis gives the value of $A_{c}^{+}$. The correlations between $A^{+}$and $\left(\widetilde{P-E)^{+}}\right.$are also labeled

LWA analysis: changing characteristics of ARs and hydrological extremes over the western US

Figure 4 displays the maps of the winter climatology of moist wave intrusions $\left(A^{+}\right), \mathrm{PR}\left(\frac{\left(A^{+}-A_{c}^{+}\right)}{A^{+}}\right)$, local sink $(\widetilde{P-E})^{+}$, and stirring scale $\left(\eta^{+}\right)$, all having been averaged over 16 CMIP5 models. As for the total wave activity, $\eta^{+2}$ resembles a local version of $l_{e}^{2}$ representing the local enhancement of mixing due to stirring/ stretching. From these maps, one can see that ARs, in a climatological sense, are characterized by large LWA, low PR, and strong large scale stirring. ${ }^{21}$ The distinct local maxima of $A^{+}$ and local PR minima collocated along the exit and the southern flank of the Pacific jet trace out the typical pathway of the Pacific ARs - the corridor of moisture transport. The small PR reveals that only a relatively small portion of wave activity participates in the hydrological cycle through condensation, embodying the primary transport function of the ARs. The large stirring length portrays the filamentary aspect of the ARs. Further, tracing backward along the AR pathway, it connects to areas of negative $(\widetilde{P-E})^{+}$, that is, the remote source regions for the downstream LWA. Note that the dominant water vapor source for the LWA $A^{+}$is the evaporation from the adjacent dry areas that are not directly considered in the budget, but indirectly accounted for through the moisture convergence term. These remote source regions identified are not artifacts of the analysis; one can discern similar source regions (tropical western Pacific, Bay of Bengal, and Arabian Sea) simply by inspecting the daily evolution of the LWA (see the Supplementary animation). When the ARs hit the west coast of the US, the resultant heavy precipitation can maintain a rather short residence time of about 1.5 days there (in contrast to 8 days over the Midwest. See Figure S4), owing to the topographic lifting at the windward flanks of the Cascades, Sierra Nevada, and Rocky Mountain ranges.

Under the RCP8.5 warming scenario, future warming reduces the HCR over the western US, as inferred from the gentler $(P-E)^{+}$vs. $A^{+}$slope in Fig. 1. On average, the HCR decreases by $\sim 15 \%$ by the late 21 st Century. This happens in the face of a much a) Dominant CWV wave activity budget terms

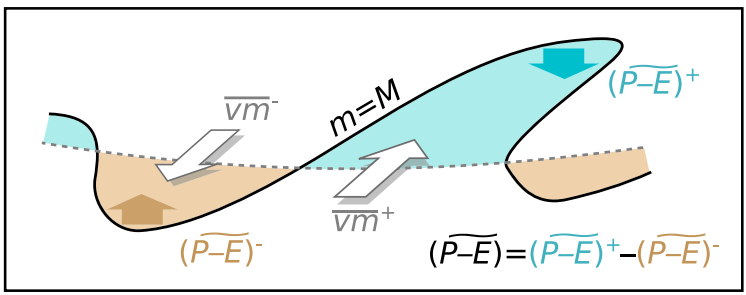

b) $(\overline{P-E})^{+}$dissipates $A^{+}$towards $A_{c}^{+}$at time scale $\mathrm{T}$

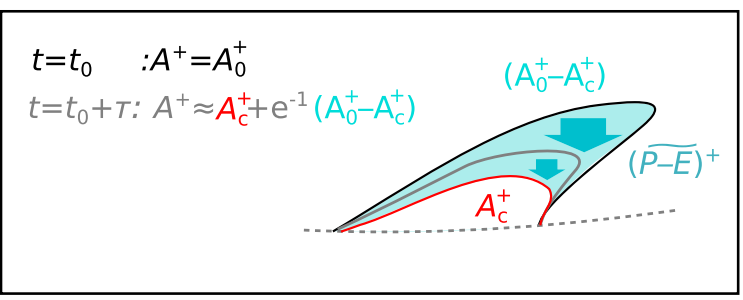

c) the participation ratio

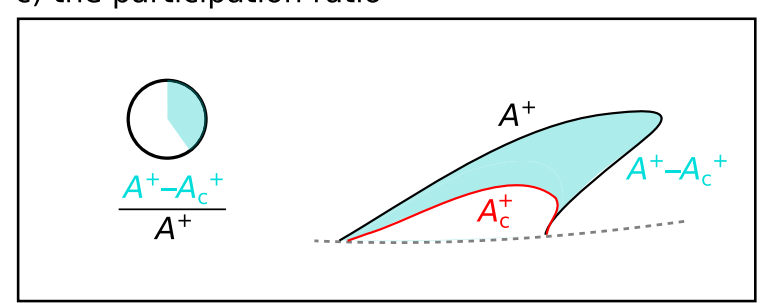

Fig. 2 Schematic for the wavy hydrological cycle. The wave component of the hydrological cycle is instigated by the meridional advection ( $\overline{v m}$, shadowed arrows) of CWV across the equivalent latitude $\left(\phi_{e}\right)$ and dissipated by the precipitation minus evaporation $(P-E)$ in the moist areas and $E-P$ in the dry areas (vertical arrows). The hydrological cycle associated with the moist intrusion can be portrayed by moist wave activity $A^{+}$, its dissipation time scale $\tau$, and participation ratio (PR) $\frac{A^{+}-A_{c}^{+}}{A^{+}}$. 

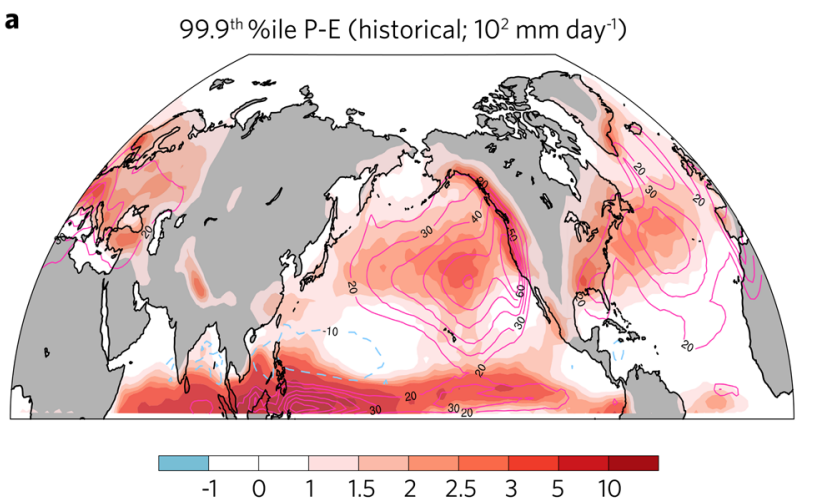

b

b $99.9^{\text {th }}$ \%ile P-E (change; $m m$ day $^{-1}$ )

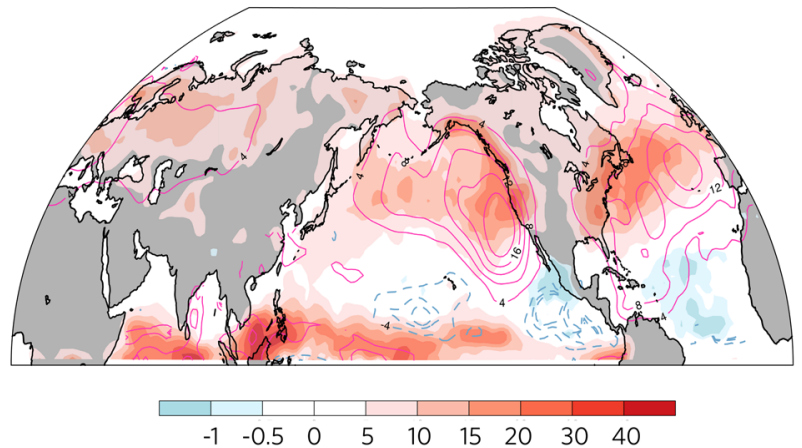

Fig. 3 Spatial correspondence between $(\widetilde{P-E})^{+}$and 99.9th percentile of $P-E$. a Climatology estimated using 2700 winter days for each model and averaged over the 16 models examined; $\mathbf{b}$ the multi-model ensemble mean difference between RCP8.5 future scenario (2070-2099) and historical (1976-2005) simulations. The features of $(\widetilde{P-E})^{+}$(contours) are equatorward displaced compared to those of the 99.9th percentile of $P-E$ (shading) because the result of the coordinate transformation is registered on the equivalent latitude, which is always equatorward to the moisture intrusion

larger increase ( 40\%) in wave activity $A^{+}$(Fig. 6b), which, being dampened by a reduction in HCR $(-15 \%$, Fig. $6 \mathrm{~d})$ and PR $(-6 \%)$, still results in $\sim 24 \%$ increase in $(\widetilde{P-E})^{+}$(Fig. 6a). The spatial distribution of the LWA changes is dominated by that of the stirring scale $\eta^{+}$(Fig. 6c), as can be inferred from the scaling of the wave activity. Compared to its background distribution, the $\eta^{+}$ anomalies over the eastern Pacific and North America are indicative of a downstream and southward extension of the total stirring scale. This is consistent with the finding that ARs that make landfall in the western US are projected to push equatorward in winter under RCP8.5 scenario compared to the present climate using conventional detection methods of ARs. ${ }^{4,6}$ If $\eta^{+}$near the US coast can be thought of as a measure of the length of the landfalling ARs, there appears to be a lengthening of the ARs there in a warmer climate. Intriguingly, the increase of $\eta^{+}$over North America is also shared by other midlatitude continents in the Northern Hemisphere (not shown); this midlatitude wide continental increase warrants further investigation, while evidence exists for the lengthening of the intermediate-scale stationary wave under the same climate change scenario. ${ }^{30}$

In view of the encouraging consensus on the sign of the changes in $A^{+}, \widetilde{(P-E)^{+}}$, and HCR among the CMIP5 models examined (stippling in Figs. 6 and 7), some confidence may be assigned to these projected hydrological changes over the west coast of the US. Also noteworthy is that, over the west coast, the change of the standard deviation of $(\widetilde{P-E})^{+}$exhibits a commensurate rate with the change of the $(\widetilde{P-E})^{+}$itself; models with a greater increase in $\left(\widetilde{P-E)^{+}}\right.$tend to have a greater increase in the standard deviation as well (Figure S5). The same may also be said for $A^{+}$.

\section{DISCUSSION}

A CWV-following analysis has been developed for the wave component of the hydrological cycle and extended to study regional hydrological cycle. The Lagrangian nature of the diagnostics reveals a tight relationship between the transformed $P-E$ and moisture anomalies, both being more normally distributed than their Eulerian counterparts. Through this relationship, the large increase of wet vs. dry disparity under a future climate change scenario can be further deciphered into a dynamical component (increase of stirring scale) and thermodynamical one (meridional gradient of the Lagrangian CWV), and a factor of HCR, the latter showing an overall weakening globally. The zonal mean CWV wave activity can be manifested in the regional hydrological features like ARs, the moisture transport function of which and the associated large HCR upon making landfall are revealed by the local CWV wave activity analysis. The enhanced stirring and the lengthening of the ARs during winter time serve as an important dynamical underpinning behind the projected robust increase of the precipitation extremes over the western states of the America under the RCP 8.5 scenario.

The robust increase in the northward water vapor wave activity and stirring scale tempts us to draw analogy to the contentious claim that Arctic sea ice melt and polar amplification of warming can give rise to greater waviness and more frequent extreme weathers. ${ }^{31-34} \mathrm{An}$ important distinction here is that the background CWV gradient is enhanced under warming for the latitudinal range examined, while the opposite is true for the surface temperature gradient associated with polar amplification. Acting on the enhanced background moisture gradient, an increase of the stirring scale $\eta^{+}$would certainly intensify the moist extremes if our climate model result is any guide. Closer inspection on the pattern of the change of wave activity $A^{+}$ strongly suggests a southward expansion and downstream extension near the exit of the Pacific jet, concomitant with the southward shift and downstream extension of the Pacific jetanother robust aspect of the winter circulation change projected by the CMIP5. ${ }^{2,35}$ Further support can be gained from the significant correlation between the inter-model spread in the changes of the zonal wind speed near the jet exit (centered at $30^{\circ}$ $\left.\mathrm{N}, 220^{\circ} \mathrm{E}\right)$ and that of $A^{+}$over the west coast $\left(30^{\circ}-50^{\circ} \mathrm{N}, 235^{\circ}-245^{\circ} \mathrm{E}\right)$ (Figure S6). The eastward extension of $A^{+}$is also in keeping with the eastward shift of the global warming-induced changes in $\mathrm{El}$ Niño teleconnections over the North Pacific and North America. ${ }^{36,37}$ Pieced together, these constitute a coherent picture that the downstream extension of the Pacific jet advects the transient disturbances and the associated hydrological variations downstream, bringing more hydrological extremes to the west coast. ${ }^{38}$ Given that this circulation change is the most important denominator for the hydrological extremes over the western US, the focus of future research should be directed towards the dynamical mechanisms of the response of the Pacific jet-wave system to climate warming. To the extent that some confidence may be garnered from the agreed Pacific circulation change among the CMIP5 models, we conjecture that the unusually wet winter experienced in the 2016/17 might be a harbinger for something more frequent to come in a warmer world.

\section{METHODS}

Formulation of CWV finite amplitude wave activity (FAWA) budget To evaluate the wave component of the hydrological cycle that has more immediate bearing on extremes, we apply the area-coordinate 
a

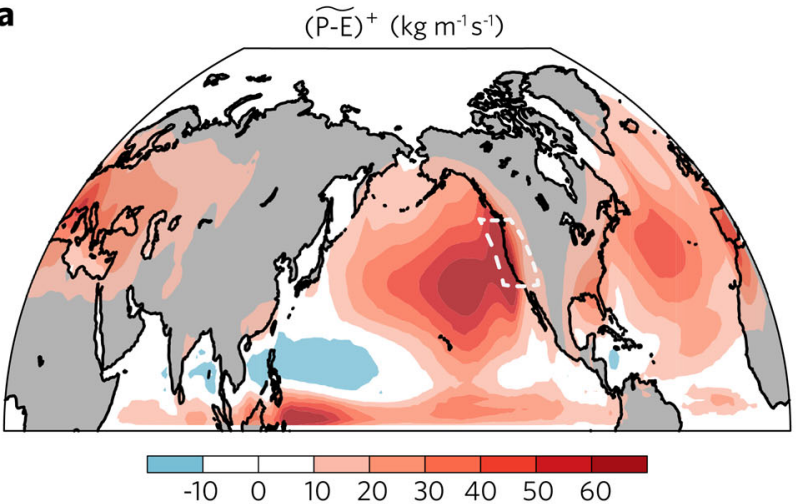

C

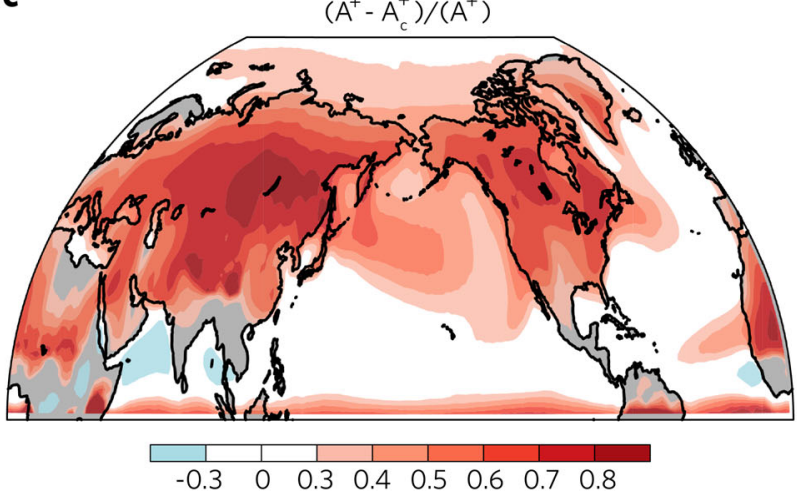

b

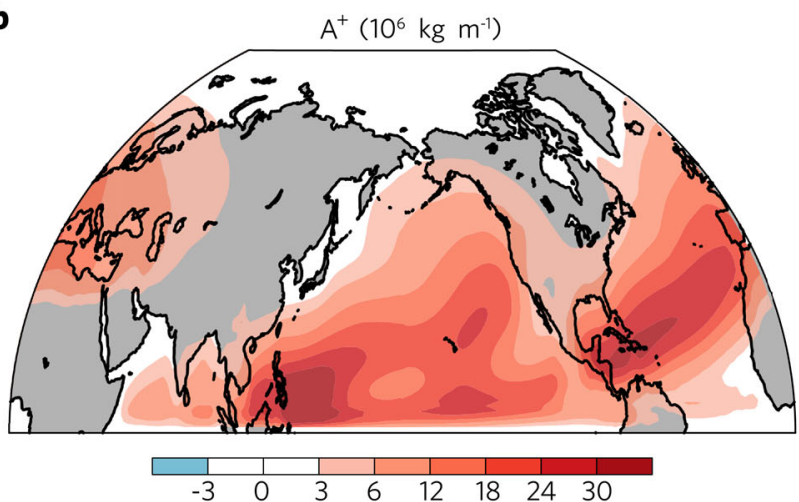

d

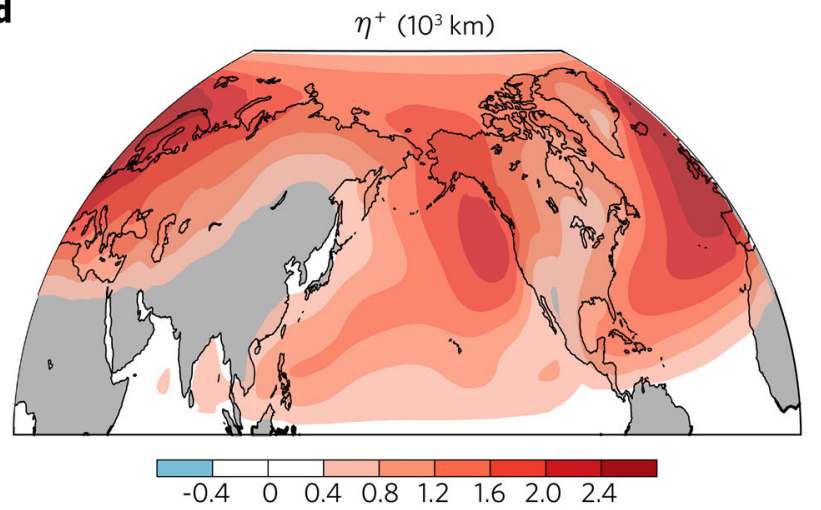

Fig. 4 Climatological distribution of quantities due to moist intrusion. Global distribution of the winter climatology of a $(\widetilde{P-E})^{+}$; $\mathbf{b} A^{+}$; $\mathbf{c}$ $\frac{\left(A^{+}-A_{c}^{+}\right)}{A^{+}}$and $\mathbf{d}$ stirring scale $\eta^{+}$based on the average of the historical simulations by 16 CMIP5 models. The white box in Fig. $5 a$ marks the area where the quantities in Fig. 1 are averaged

transformation of Nakamura (e.g., ref. ${ }^{21,39-41}$ ) to the CWV budget equation $^{42}$

$\frac{\partial m}{\partial t}+\boldsymbol{v}_{\psi} \cdot \nabla m=E-P-\nabla \cdot\left(\boldsymbol{v}_{X} m\right)$

where $m$ is CWV, $v=\frac{\frac{1}{p_{5}} q V \mathrm{~d} p}{1 p_{5}} q \mathrm{~d} p$ is water vapor weighted horizontal velocity with $\boldsymbol{v}_{\psi}$ and $\boldsymbol{v}_{X}$ being its rotational and divergent components, respectively. $P$ and $E$ are precipitation and evaporation. The aforementioned area-coordinate transformation is defined as

$\Delta \mathcal{A}() \equiv-\frac{1}{2 \pi a \cos \phi_{e}}\left(\iint_{m<M}() d S-\iint_{\phi>\phi_{e}}() d S\right)$,

where $M$ (the upper case is used to emphasize its Lagrangian property) is the value of an instantaneous, wavy CWV contour with an equivalent latitude $\phi_{e}$, which itself is determined uniquely by the requirement that the area enclosed poleward of the $M$ contour equals the area poleward of latitude $\phi_{e}$ in the Northern Hemisphere (see Fig. 8). Effectively, a variable subject to the transformation (2) gives the normalized difference (by $2 \pi a \cos \phi_{e}$ ) between its area integration over the green areas and that over the brown areas districted by the contour $M$. The resultant equation from the transformation is the budget equation for the CWV FAWA:

$\frac{\partial A}{\partial t}-\overline{v_{\psi}^{\prime} m^{\prime}}=\left[\widetilde{(E-P)}-\nabla \cdot \widetilde{\left(\boldsymbol{v}_{X} m\right)}\right]$,

where overline indicates zonal mean and prime the deviation from the zonal mean, and ${ }^{\sim}$ has replaced $\Delta \mathcal{A}()$ for the compactness of the equation. In the limit of small perturbation, one can show that $A \rightarrow-\left(\frac{1}{a} \frac{\partial m}{\partial \phi}\right)^{-1} \frac{m \prime^{2}}{2}$ $\left(\mathrm{see}^{40}\right)$. Therefore, FAWA represents the second moment of the CWV variability. As illustrated in Fig. 8 , the budget of $A$ is balanced by the eddy CWV flux across the equivalent latitude $\phi_{e}$, the divergent flux across the $M$ contour, and the wave activity sink term $(\widetilde{E-P})$. The detailed derivation from (1) to (3) can be found in Lu et al. ${ }^{21}$ With $\overline{v_{\psi}^{\prime} m^{\prime}}$ and $\nabla \cdot \overline{\left(\boldsymbol{v}_{x} m\right)}$ collapsing into $\nabla \cdot \widetilde{(v m)}$, Eq. (3) can also be written as

$\frac{\partial A}{\partial t}=[\widetilde{(E-P)}-\nabla \cdot \widetilde{(v m)}]$

$(\widetilde{E-P})$ is negative definite and $-\nabla \cdot \widetilde{(v m)}$ is positive definite, representing the major sink and source of the FAWA, respectively. The main advantage of the transformed CWV budget is its simplicity and amenability to interpretation, in comparison to the cumbersome Eulerian expression of moisture variance budget. The novelty of $(P-E)$ is that it represents the dry vs. wet disparity of the hydrological cycle, or the zonally anomalous $P$ $-E$, along each (equivalent) latitude.

The limitation in the temporal and vertical resolution of the CMIP5 data output prevents us from conducting a complete budget analysis for CWV FAWA using equations above. Instead, this study focuses on the emergent empirical relationship between FAWA and $(P-E)$ and the resultant simple scaling for the latter.

As found in Lu et al. ${ }^{21}$ in an aqua-planet model, there also exists in the real world a tight linear relationship between the sink term and FAWA itself:

$(\widetilde{P-E}) \sim \frac{A}{\tau}$

with $\tau$ providing the measure for the time scale at which $(\widetilde{P-E})$ acts to dissipate the wave activity towards a wave-free state $(A=0)$. Through this relation, the conceptual framework for interpreting the global hydrological cycle $\left(\tau=\frac{[m]}{|\mathrm{P}|}\right.$ with the square bracket for a global integration) can be carried over to the the wavy component of the hydrological cycle. Relation (5) also affords a simple scaling for the change of $(P-E)$ under climate 

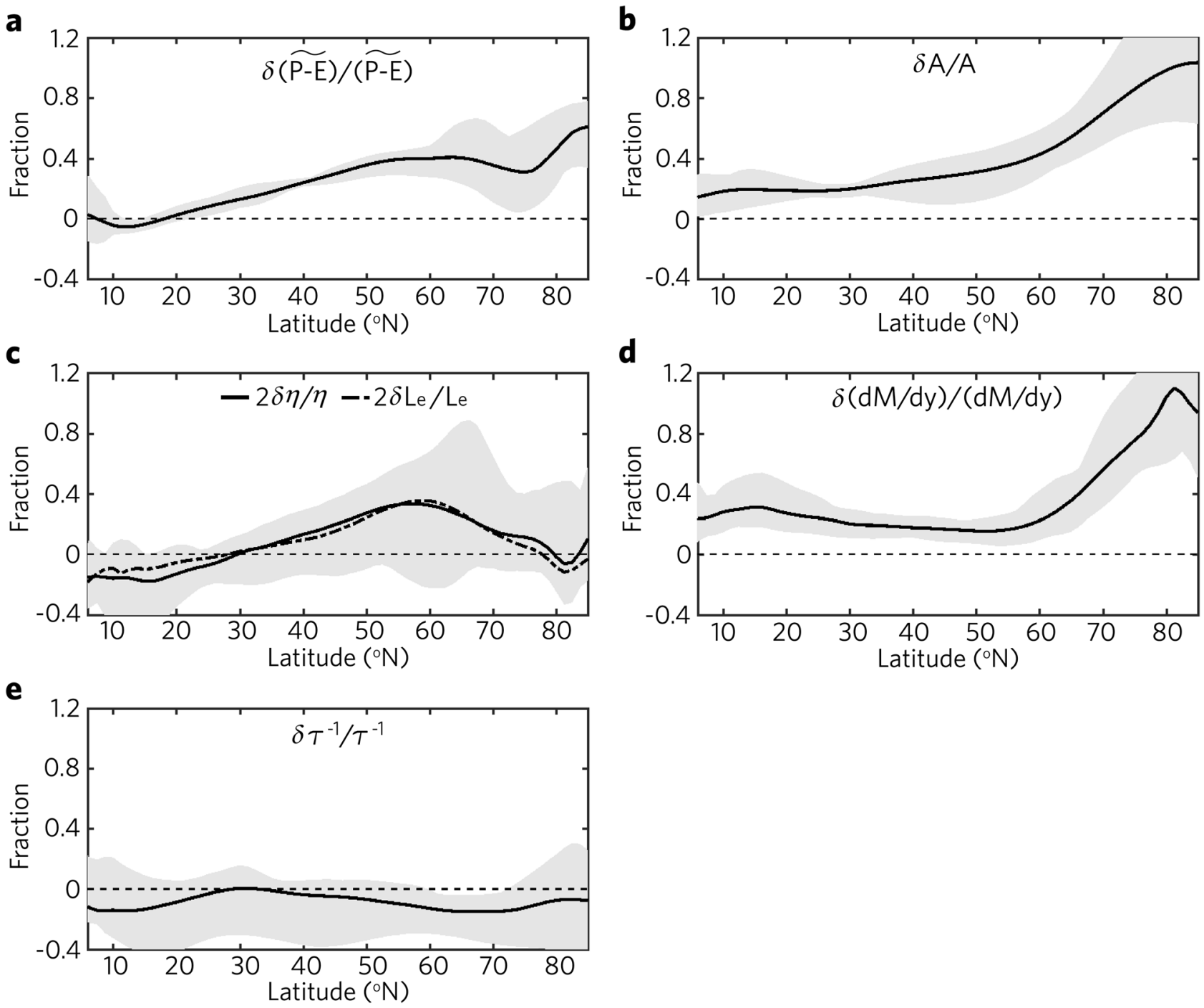

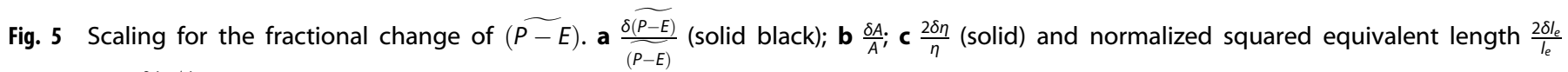
(dashed); $\mathbf{d} \frac{\delta \mathrm{d} M / \mathrm{dy}}{\mathrm{d} M / \mathrm{dy}} ;$ and $\mathbf{e} \frac{\delta \tau^{-1}}{\tau^{-1}}$. Shading indicates the intermodel spread of the corresponding variable

perturbations:

$\frac{\delta(\widetilde{P-E})}{(\widetilde{P-E})} \approx \frac{\delta A}{A}+\frac{\delta \tau^{-1}}{\tau^{-1}} \approx \frac{\frac{\delta \partial M}{\partial y}}{\frac{\partial M}{\partial y}}+\frac{2 \delta \eta}{\eta}+\frac{\delta \tau^{-1}}{\tau^{-1}}$,

with $\delta$ denoting the change of the variable in question. The second equality has used the scaling for the wave activity: $A \sim-\frac{1}{2} \eta^{2} \frac{\partial M}{\partial y}$, where $\eta$ is the meridional displacement of the contour $M$ from its equivalent latitude, resembling the concept of mixing length.

Local CWV FAWA (LWA) cycle and its scaling

The definition of the CWV FAWA can be extended to be longitudedependent (i.e., local CWV FAWA or LWA) by applying line-integral transformations to $m$ at each longitude $\lambda\left({ }^{22}\right.$; and $\left.{ }^{43}\right)$ :

$A^{-}\left(\lambda, \phi_{e}\right)=\frac{a}{\cos \phi_{e}} \int_{m \leq M, \phi \leq \phi_{e}} m(\lambda, \phi) \cos \phi d \phi$,

$A^{+}\left(\lambda, \phi_{e}\right)=\frac{a}{\cos \phi_{e}} \int_{m \geq M, \phi \geq \phi_{e}} m(\lambda, \phi) \cos \phi d \phi$.

At each longitude and with respect to a given equivalent latitude $\phi_{e}$, the integration interval is defined by the equatorward latitudinal displacement of $M$ with respect to $\phi_{e}$ (i.e., $m \leq M, \phi \leq \phi_{e}$ ) for a dry intrusion and the poleward displacement of $M$ (i.e., $m \geq M, \phi \geq \phi_{e}$ ) for a moist intrusion; the result of the integration is reported at point $\left(\lambda, \phi_{e}\right)$. Therefore, the resultant LWA has the same dimension and coordinates as the original data. Transformations (7) and (8) will be denoted as $\mathcal{L}^{-}$and $\mathcal{L}^{+}$for dry $\left(A^{-}\right)$and moist $\left(A^{+}\right)$LWA, respectively. Large $A^{+}$is often related to AR-like features and thus makes it an ideal metric for ARs or moist conveyor belts. Its corresponding wave activity sink is

$$
\begin{gathered}
(\widetilde{P-E})^{+}\left(\lambda, \phi_{e}\right) \equiv \mathcal{L}^{+}[P(\lambda, \phi)-E(\lambda, \phi)] \equiv \\
\frac{a}{\cos \phi_{e}} \int_{m \geq M, \phi \geq \phi_{e}}[P(\lambda, \phi)-E(\lambda, \phi)] \cos \phi d \phi,
\end{gathered}
$$

representing the hydrological cycle through phase change in the moist sector of a cyclone. $(P-E)^{+}$captures the higher moment of the hydrological cycle variability than the mean of $P-E$, so it links more tightly to the hydrological extremes.

Similar to the relationship between $(\widetilde{P-E})$ and $A$, significant linear relationship also exists between $(P-E)^{+}$and $A^{+}$(see Fig. 1), providing a basis for scaling $(P-E)^{+}$with the information of LWA. The significant linear relationship between $A^{+}\left(\lambda, \phi_{e}\right)$ and $(P-E)^{+}\left(\lambda, \phi_{e}\right)$ is elucidated in Figure S2, showing ubiquitous and significant correlations almost everywhere between their daily time series in winter (December-JanuaryFebruary). Since now $A^{+}$is computed from the total CWV $(m)$, an intercept 
a
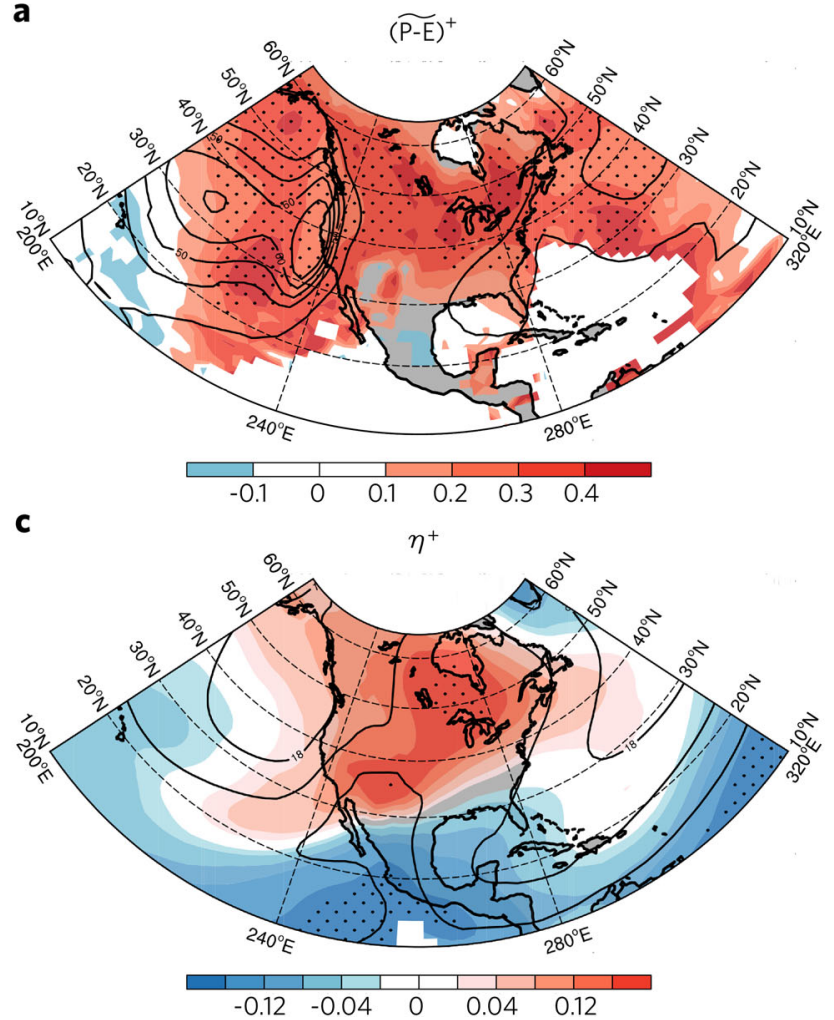

b
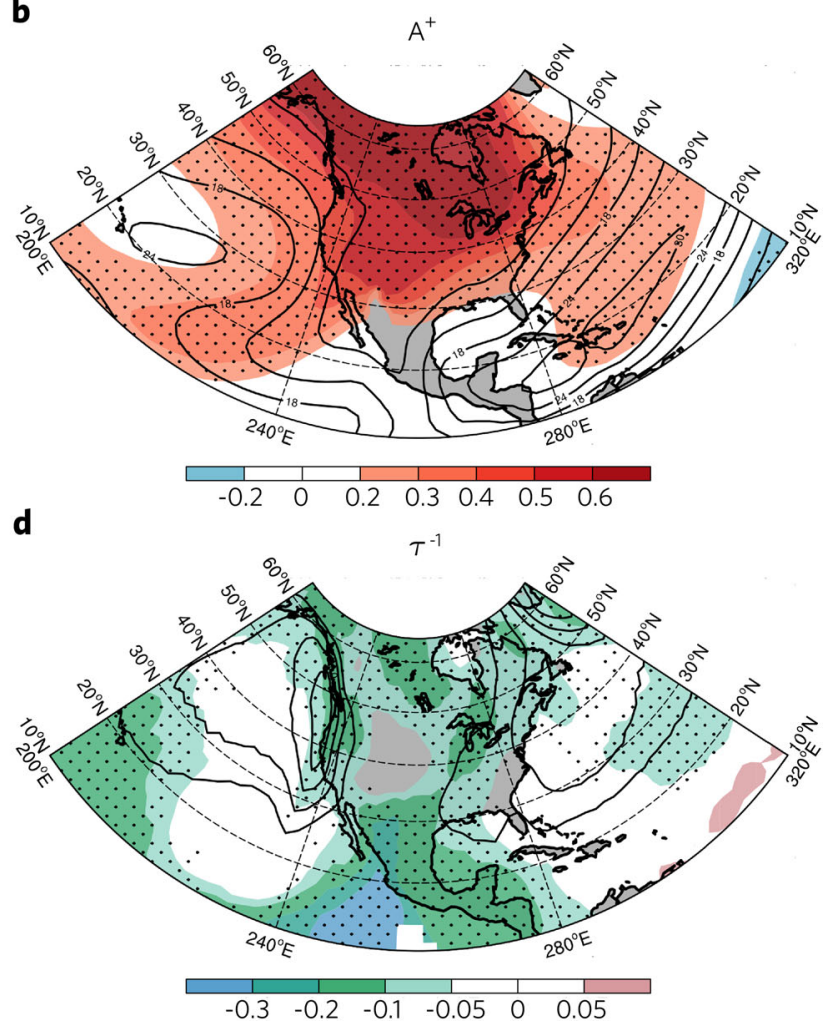

Fig. 6 Distribution of the scaling factors for $(\widetilde{P-E})^{+}$. The fractional changes in $\mathbf{a}(\widetilde{P-E})^{+} ; \mathbf{b} A^{+} ; \mathbf{c} \eta^{+}$; and $\mathbf{d}$ and $\tau^{-1}$ with the corresponding background climatology overlaid as contours. The stippling indicates regions where $>80 \%$ of the models agree on the sign of the change

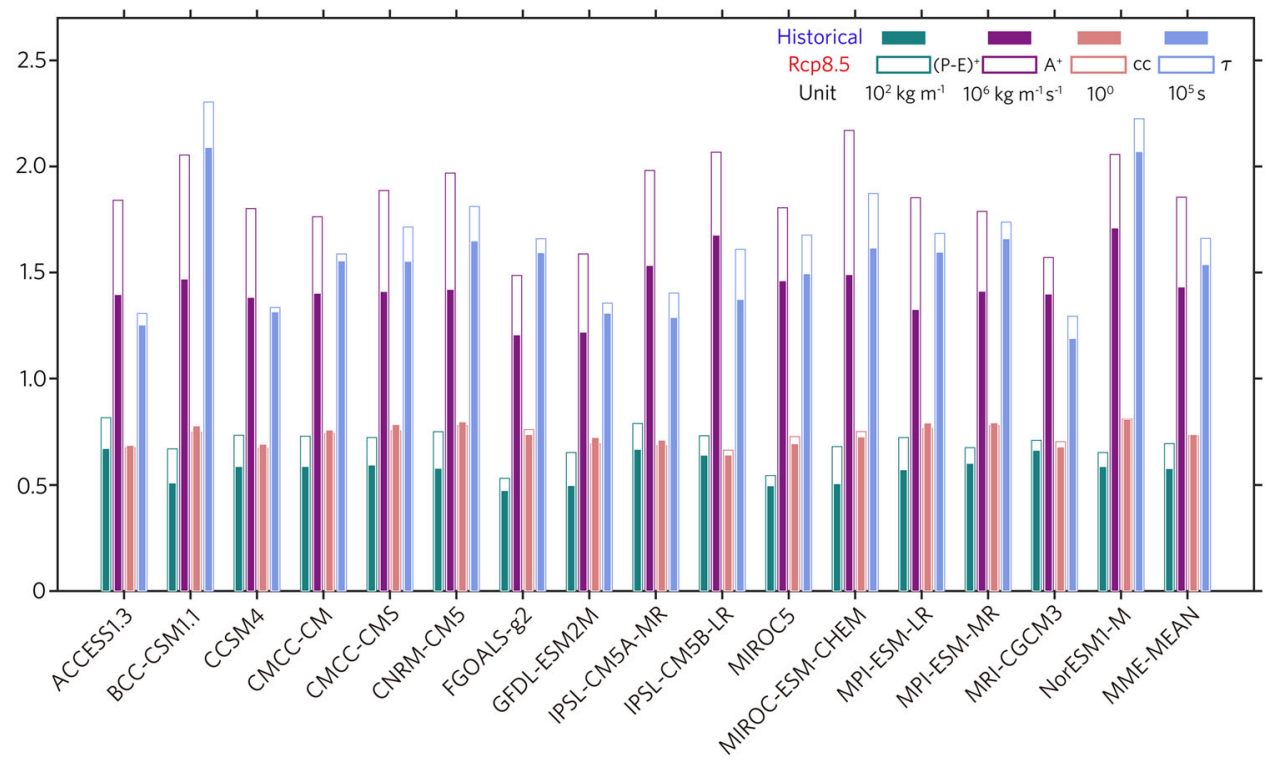

Fig. 7 Statistics of $\widetilde{(P-E})^{+}$and $A^{+}$over the west coast of the US. All quantities $\left(A^{+}, \widetilde{(P-E)^{+}}\right.$, their correlation coefficient, and residence time $\tau)$ are averaged over the west coast of the US $\left(30^{\circ}-45^{\circ} \mathrm{N}, 235^{\circ}-245^{\circ} \mathrm{E}\right)$. The filled bars are for the historical values and open bars for the RCP8.5 values

$A_{c}^{+}$ensues in the regression relationship between $A^{+}$and $\widetilde{(P-E)^{+}}$:

$(\widetilde{P-E})^{+} \approx \frac{\left(A^{+}-A_{c}^{+}\right)}{\tau}$.

$A_{c}^{+}$is generally smaller than $A^{+}$and can be thought of as the water vapor holding capacity of the atmosphere prior to saturation. Therefore, Eq. (10) offers a new angle to interpret the hydrological cycle in the moist areas: $P-E$ is operating as such to restore the CWV towards the capacity level $A_{c}^{+}$at time scale $\tau$, which itself is the regression slope. The physical meaning of $A_{c}^{+}$can be further deciphered by scaling $A^{+}$as $-\frac{1}{2} \eta^{+2} \frac{\partial M}{\partial y}$ and $A_{c}^{+}$as $-\frac{1}{2} \eta_{c}^{+2} \frac{\partial M}{\partial y}$, respectively. As such, $\eta_{c}^{+}$assumes a meaning of "mean free path" of an air parcel within which the air parcel can travel freely poleward with the humidity content conserved, while $\eta^{+}$ represents the actual distance traveled by the air parcels and the $\eta^{+}>\eta_{c}^{+}$ 


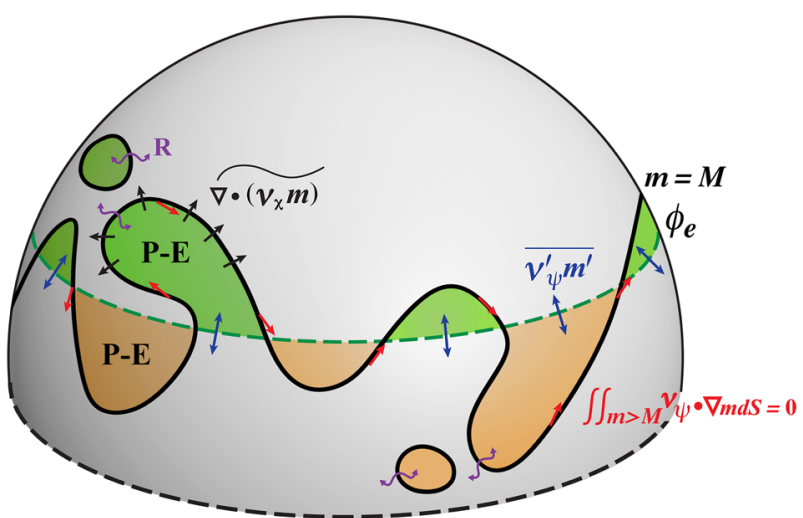

Fig. 8 Schematic for the wave activity and its budget. The wavy solid curve denotes a contour of CWV $(m)$. In the green (brown) areas demarcated between $m$ contour and the corresponding equivalent latitude (dashed green line), $\mathrm{m}$ is greater (less) than $\mathrm{M}$. The budget for wave activity $A$ is comprised of a flux of CWV anomalies $\left(m^{\prime}=m-M\right)$ across the equivalent latitude $\left(\phi_{e}\right)$ by the rotational flow $\overline{v_{\psi}^{\prime} m^{\prime}}$, a flux across the $M$ contour due to the divergent flow $-\nabla \cdot \widetilde{\left(\boldsymbol{v}_{X} m\right)}$, and a sink term $\widetilde{(E-P)} . \mathrm{R}$ term is a small residual due to numerical dissipation

part is the distance over which condensation takes place. Effectively, only the $A^{+}-A_{c}^{+}$portion of $A^{+}$participates in the hydrological cycle and hence is subject to the dissipation by $P-E$. Consequently, the ratio of $A^{+}-A_{c}^{+}$to $A^{+}$can be defined as the PR of the local hydrological cycle (see Fig. 4c).

The relationship (10) leads to a simple scaling for the fractional change of hydrological cycle in the moist sectors:

$$
\begin{gathered}
\frac{\delta(P-E)^{+}}{(P-E)^{+}} \approx \frac{\delta\left(\frac{A^{+}-A_{C}^{+}}{A^{+}}\right)}{\left(\frac{A^{+}-A_{C}^{+}}{A^{+}}\right)}+\frac{\delta A^{+}}{A^{+}}+\frac{\delta \tau^{-1}}{\tau^{-1}} \approx \\
\frac{\delta\left(\frac{A^{+}-A_{C}^{+}}{A^{+}}\right)}{\left(\frac{A^{+}-A_{C}^{+}}{A^{+}}\right)}+\frac{\frac{\partial M}{\partial M}}{\partial y}+\frac{2 \delta \eta}{\eta}+\frac{\delta \tau^{-1}}{\tau^{-1}} .
\end{gathered}
$$

Within this framework, the fractional change of $\widetilde{\frac{\delta(P-E)^{+}}{}}$can be interpreted as the sum of the fractional changes in $P\left(R^{-}, E_{\text {moist }}^{+}\right.$wave activity, and HCR.

\section{Normalized squared equivalent length}

Provided that the form of the small-scale diffusion for a tracer is known, the equivalent length measuring the enhancement of the small-scale diffusion via the stirring and stretching by the resolved eddies can be computed directly based on the geometry of the tracer evolution (e.g., $\left.{ }^{41,44}\right)$. Without loss of generality, applying the area-coordinate transformation $\Delta \mathcal{A}$ to the regular diffusion of $\mathrm{CWV} \kappa \nabla^{2} m$ yields

$\Delta \mathcal{A}\left[\kappa \nabla^{2} m\right] \approx-\kappa \frac{\left\langle|\nabla m|^{2}\right\rangle_{M}}{\left(\frac{\partial M}{\partial y}\right)^{2}} \frac{\partial M}{\partial y}=-l_{e}^{2} \kappa \frac{\partial M}{\partial y}$,

where the subscripted angle bracket denotes the average along the $M$ contour, the non-dimensional metric $l_{e}^{2}=\frac{\left\langle|\nabla m|^{2}\right\rangle_{M}}{\left(\frac{\partial M}{2}\right.}$ is the normalized squared equivalent length. As elucidated $\operatorname{in}^{\left(\frac{\partial M}{\partial y}\right)^{2}}(12), l_{e}^{2}$ quantifies the amplification effect on $\kappa$ by the model-resolved stirring and stretching as FAWA becomes the subject of dissipation. The square factor stems from the dual effects of stretching-both elongating the tracer interface and sharpening the gradient normal to the tracer contour. See Appendix B in Lu et al. ${ }^{20}$ for the exact derivation of (12). Its fractional change under RCP8.5 scenario agrees well with twice the fractional change of $\eta$ (Fig. 5c), further vindicating the role of the identified dynamical component in the change of the zonally anomalous hydrological cycle.

\section{Data and code availability}

CMIP5 model output is available from the Earth System Grid Federation (ESGF) Peer-to-peer system (https://esgf-node.IInl.gov/projects/cmip5). The
Matlab code for computing CWV FAWA and LWA and their budget terms can be obtained from the lead author upon request.

\section{ACKNOWLEDGEMENTS}

This study was supported by the US Department of Energy Office of Science Biological and Environmental Research (BER) as part of the Regional and Global Climate Modeling program. DX was supported by the National Nature Science Foundation of China (Grant 41621005), Jiangsu Collaborative Innovation Center for Climate Change, and jointly supported by the China Scholarship Council. We acknowledge the World Climate Research Programme's Working Group on Coupled Modeling, which is responsible for CMIP, and we thank the climate modeling groups for producing and making available their model output. GC is supported by the DOE grant DE-SC0016117.

\section{AUTHOR CONTRIBUTIONS}

J.L. came up with the original ideas, developed the FAWA analysis for hydrological cycle, and led the data analysis and the writing of the manuscript. D.X. contributed equally to the study by conducting all the analysis reported in the manuscript and producing all the figures; Y.G. prepared all the data sets used in the study and helped with the pre-analysis of the data; G.C., R.Y.L., and P.S. assisted with the interpretation and presentation of the scientific results and participated in the writing and revision of the manuscript.

\section{ADDITIONAL INFORMATION}

Supplementary information accompanies the paper on the npj Climate and Atmospheric Science website (https://doi.org/10.1038/s41612-018-0017-9).

Competing interests: The authors declare no competing financial interests.

Publisher's note: Springer Nature remains neutral with regard to jurisdictional claims in published maps and institutional affiliations.

\section{REFERENCES}

1. Trenberth, K. E. et al. The changing character of precipitation. Bull. Am. Meteorol. Soc. 84, 1205-1217 (2003).

2. Gao, Y. et al. Dynamical and thermodynamical modulations on future changes of landfalling atmospheric rivers over western North America. Geophys. Res. Lett. https://doi.org/10.1002/2015GL065435 (2015).

3. Warner, M., Mass, C. F. \& Salathe, E. P. Changes in winter atmospheric rivers along the North American west coast in CMIP5 climate models. J. Hydrometeor. 16, 118-128 (2015).

4. Payne, A. E. \& Magnusdottir, G. An evaluation of atmospheric rivers over the North Pacific in CMIP5 and their response to warming under RCP 8.5. J. Geophys. Res. 120, 11, 173-11, 190 (2015).

5. Hagos, S. et al. A projection of changes in landfalling atmospheric river frequency and extreme precipitation over western North America from the Large Ensemble CESM simulations. Geophys. Res. Lett. 43, 1357-1363 (2016).

6. Shields, C. A. \& Kiehl, J. T. Atmospheric river landfall-latitude changes in future climate simulations. Geophys. Res. Lett. 43, 8775-8782 (2016).

7. Ralph, F. M. et al. Flooding on California's Russian River: the role of atmospheric rivers. Geophys. Res. Lett. 33, L13801 (2006).

8. Neiman, P. J. et al. Flooding in western Washington: the connection to atmospheric rivers. J. Hydrometeor. 12, 1337-1358 (2011).

9. Emori, S. \& Brown., S. J. Dynamic and thermodynamic changes in mean and extreme precipitation under changed climate. Geophys. Res. Lett. https://doi.org/ 10.1029/2005GL023272 (2005).

10. O'Gorman, P. A. \& Schneider, T. The physical basis for increase in precipitation extremes in simulations of $21^{\text {st }}$-centruy climate change. PNAS 106, 14473-14477 (2009).

11. Lu, J. et al. The robust dynamical contribution to precipitation extremes in idealized warming simulations across model resolutions. Geophys. Res. Lett. 41, 2971-2978 (2014).

12. Collins, M. \& Knutti, R. et al. in Climate Change 2013: The Physical Science Basis-Working group I. In: Contribution to the fifth assessment report of the intergovernmental panel on climate change (eds Stocker T. F. et al.) Ch. 12 (Cambridge University Press, Cambridge and New York, NY, 2013).

13. Toreti, A. et al. Projections of global changes in precipitation extremes from Coupled Model Intercomparison Project Phase 5 models. Geophys. Res. Lett. 40, 4887-4892 (2013). 
14. Kharin, V. V. \& Zwiers, F. W. Estimating extremes in transient climate change simulations. J. Clim. 18, 1156-1173 (2005).

15. Gershunov, A. et al. Assessing the climate-scale variability of atmospheric rivers affecting western North America. Geophys. Res. Lett. 44, 7900-7908 (2017).

16. Allen, M. R. \& Ingram, W. J. Constraints on future changes in the hydrological cycle. Nature 419, 224-228 (2002).

17. Held, I. M. \& Soden, B. J. Robust response of the hydrological cycle to global warming. J. Clim. 19, 5686-5699 (2006).

18. Stephens, g. L. \& Hu, Y. Are climate-related changes to the character of globalmean precipitation predictable? Env. Res. Lett. https://doi.org/10.1088/1748-9326/ 5/2/025209 (2010)

19. Nakamura, N. Modified Lagrangian-mean diagnostics of the stratospheric polar vortices. Part I: Formulation and analysis of GFDL SKYHI GCM. J. Atmos. Sci. 52, 2096-2108 (1995).

20. Lu, J. et al. Towards the dynamical convergence on the jet stream in aquaplanet AGCMs. J. Clim. 28, 6763-6782 (2015).

21. Lu, J. et al. Examining the hydrological variations in an aquaplanet world using wave activity transformation. J. Clim. 30, 2559-2576 (2017).

22. Huang, C. S. Y. \& Nakamura, N. Local finite-amplitude wave activity as a diagnostic of anomalous weather events. J. Atmos. Sci. 73, 211-229 (2016).

23. Taylor, K. E. et al. An overview of CMIP5 and the experiment design. Bull. Am. Meteor. Soc. 93, 485-498 (2012).

24. Lu, J. Vecchi, G., \& Reichler, T. Expansion of the Hadley cell under global warming Geophys. Res. Lett. 34, https://doi.org/10.1029/2006GL028443 (2007).

25. Lu, J., Chen, G. \& Frierson, D. Response of the zonal mean atmospheric circulation to El Nino versus global warming. J. Clim. 21, 5835-5851 (2008).

26. Lorenz, D. J. \& DeWeaver, E. T. The response of the extratropical hydrological cycle to global warming. J. Clim. 20, 3470-3484 (2007).

27. Laliberté, F. et al. Constrained work output of the moist atmospheric heat engine in a warming climate. Science 347, 540-543 (2015).

28. Kay, J. E. et al. The Community Earth System Model (CESM) Large Ensemble Project: a community resource for studying climate change in the presence of internal climate variability. BAMS 96, 1333-1349 (2015).

29. Singh, M. S. \& O'Gorman, P. A. Upward shift of the atmospheric general circulation under global warming: theory and simulations. J. Atmos. Sci. 25, 8259-8276 (2013).

30. Simpson, I. et al. Cause of change in Northern Hemisphere winter meridional winds and regional hydroclimate. Nat. Clim. Change 6, 65-70 (2016).

31. Francis, J. A. \& Vavrus, S. J. Evidence for a wavier jet stream in response to rapid Arctic warming. Environ. Res. Lett. 10, https://doi.org/10.1088/1748-9326/10/1/ 014005 (2015).

32. Tang, Q. et al. Cold winter extremes in northern continents linked to Arctic sea ice loss. Environ. Res. Lett. 8, https://doi.org/10.1088/1748-9326/8/1/014036 (2013).

33. Cohen, J. et al. Recent Arctic amplification and extreme mid-latitude weather. Nat. Geosci. 7, 627-637 (2014)

34. Screen, J. A. \& Simmonds, I. Exploring links between Arctic amplification and midlatitude weather. Geophys. Res. Lett. 40, 959-964 (2013).
35. Neelin, D. J. et al. California winter precipitation change under global warming in the Coupled Intercomparison Project Phase 5 Ensemble. J. Clim. 26, 6238-6256 (2013)

36. Xie, S.-P. et al. Global warming pattern formation: Sea surface temperature and rainfall. J. Clim. 23, 966-986 (2010).

37. Zhou, Z.-Q. et al. Global warming-induced changes in El Niño teleconnections over the North Pacific and North America. J. Clim. 27, 9050-9064 (2014).

38. Branstator, G. \& Teng, H. Tropospheric waveguide teleconnections and their seasonality. J. Atmos. Sci. 74, 1513-1532 (2017).

39. Nakamura, N. \& Solomon, A. Finite-amplitude wave activity and mean flow adjustments in the atmospheric general circulation. Part I: Quasigeostrophic theory and analysis. J. Atmos. Sci. 67, 3967-3983 (2010).

40. Nakamura, N. \& Solomon, A. Finite-amplitude wave activity and mean flow adjustments in the atmospheric general circulation. Part II: Analysis in the isentropic coordinate. J. Atmos. Sci. 68, 2783-2799 (2011).

41. Chen, G. \& Plumb, A. R. Effective Isentropic diffusivity of tropospheric transport. J. Atmos. Sci. 71, 3499-3520 (2014).

42. Trenberth, K. E. \& Guillemot, C. J. Evaluation of the global atmospheric moisture budget as seen from analysis. J. Clim. 8, 2255-2272 (1995).

43. Chen, G. et al. Local finite-amplitude wave activity as an objective diagnostic of midlatitude extreme weather. Geophys. Res. Lett. 42, 10952-10960 (2015).

44. Nakamura, N. Two-dimensional mixing, edge formation, and permeability diagnosed in an area coordinate. J. Atmos. Sci. 53, 1524-1537 (1996).

45. Zhu, Y. \& Newell, R. E. A proposed algorithm for moisture fluxes for atmospheric rivers. Mon. Wea. Rev. 126, 725-735 (1998).

46. Carlson, T. N. Airflow through midlatitude cyclones and the comma cloud patterns. Mon. Wea. Rev. 108, 1498-1509 (1980).

47. Boutle, I. A., Belcher, S. E. \& Plant, R. S. Moisture transport in mid-latitude cyclones. Q. J. Roy. Meteor. Soc. 136, 1-15 (2011).

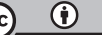

Open Access This article is licensed under a Creative Commons Attribution 4.0 International License, which permits use, sharing, adaptation, distribution and reproduction in any medium or format, as long as you give appropriate credit to the original author(s) and the source, provide a link to the Creative Commons license, and indicate if changes were made. The images or other third party material in this article are included in the article's Creative Commons license, unless indicated otherwise in a credit line to the material. If material is not included in the article's Creative Commons license and your intended use is not permitted by statutory regulation or exceeds the permitted use, you will need to obtain permission directly from the copyright holder. To view a copy of this license, visit http://creativecommons. org/licenses/by/4.0/.

(c) The Author(s) 2018 